\title{
Sensing, Storing and Computing - Spinelectronics as a Universal Platform
}

Günter Reiss and Andreas Hütten

Bielefeld University, Physics Department

Universitätsstraße 25, 33615 Bielefeld, Germany

\section{Introduction}

Within the last decade, the development of micro- and nanoelectronics has led to a large variety of products used in almost any field of everyday's life. Whereas the most prominent examples are processors and storing devices in computers, nowadays electronics including sensors is integrated more and more in cars, machines and in handheld devices such as mobile phones or digital cameras.

For this wide range of applications, a large number of physical effects and technological processes are used to realize sensor devices: Commercial sensor devices are up to now based on various effects such as Hall effect or Anisotropic Magneto Resistance (AMR).

Following the discoveries of a large magnetoresistance (Giant Magneto Resistance, GMR) in purely metallic thin film systems [1, 2] and an even larger Tunneling Magneto Resistance (TMR) in tunneling junctions with insulating barriers $[3,4]$ by german, french, american and japanese researchers, these effects were transferred rapidly to applications in read heads for hard disk drives. Additionally, however, they can provide both flexible sensing devices as well as nonvolatile storing and computing. The emerging field of research and development was named "Magneto- or Spinelectronics". As sketched in Figure 1, potential applications developed also in the field of communication via the generation of microwaves for, e.g., on chip information transport, in optics and quantum computing. The importance of this development is underlined by the nobel prize in physics awarded in 2007 to Peter Grünberg and Albert Fert for their discovery of the Giant Magneto Resistance effect [6].

\section{Spin dependent transport}

Giant Magnetoresistance (GMR), Tunneling Magnetoresistance (TMR)

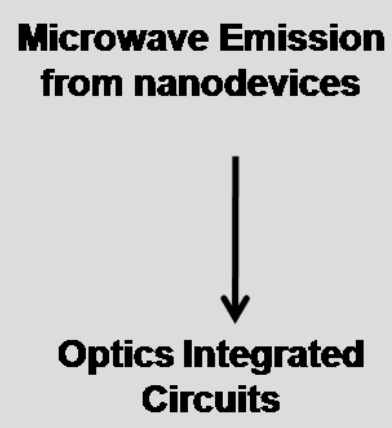

Microwave Emission
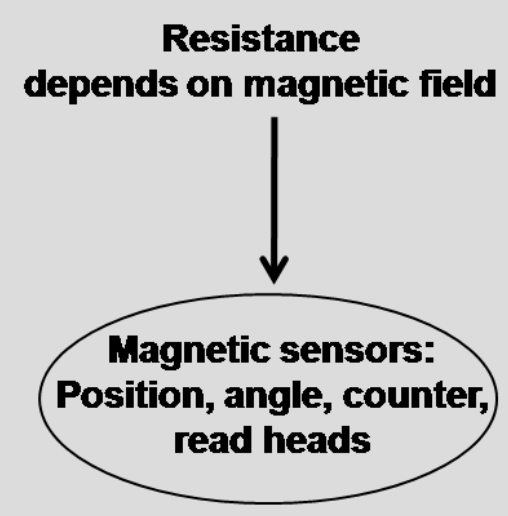

has two stable states

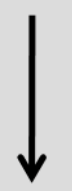

Non volatile Memory

Field programmable

Logic Gate Arrays

Figure 1: A classification of magneto- and spinelectronics for applications in sensing, storing, computing and communication.

There is of course a large market potential for such a wide variety of applications realized on one technological platform: Nonvolatile storing in RAMs has a market size of more than 100 billion $€$ per year, read heads for hard disk drives are in the range of more than 50 billion $€$ p.a.. Compared to these numbers, the market for sensors with around 5 to 10 billion $€$ p.a. is comparably small, but frequently these sensors are key components for a better performance of complete systems. In this contribution, we concentrate on the sensor applications of these new devices. 


\section{Giant- or Tunneling Magnetorsistance as the basic effect for applications}

The basic effect of for sensing and storing is illustrated in Figure 2: Two thin ferromagnetic films are separated by a thin nonmagnetic metal or insulator. Then the electrical resistance $\mathrm{R}$ of this arrangement depends strongly on the relative orientations of the magnetizations of the two ferromagnets. In most material combinations, the resistance in the parallel configuration $\left(R_{\uparrow}\right)$ is much smaller than in the antiparallel one $\left(R_{\uparrow \downarrow}\right)$. The maximum resistance change of the effect is then defined as $\Delta R=\left(R_{\uparrow \downarrow}-R_{\uparrow \uparrow}\right) / R_{\uparrow \uparrow}$ (see Figure 2).

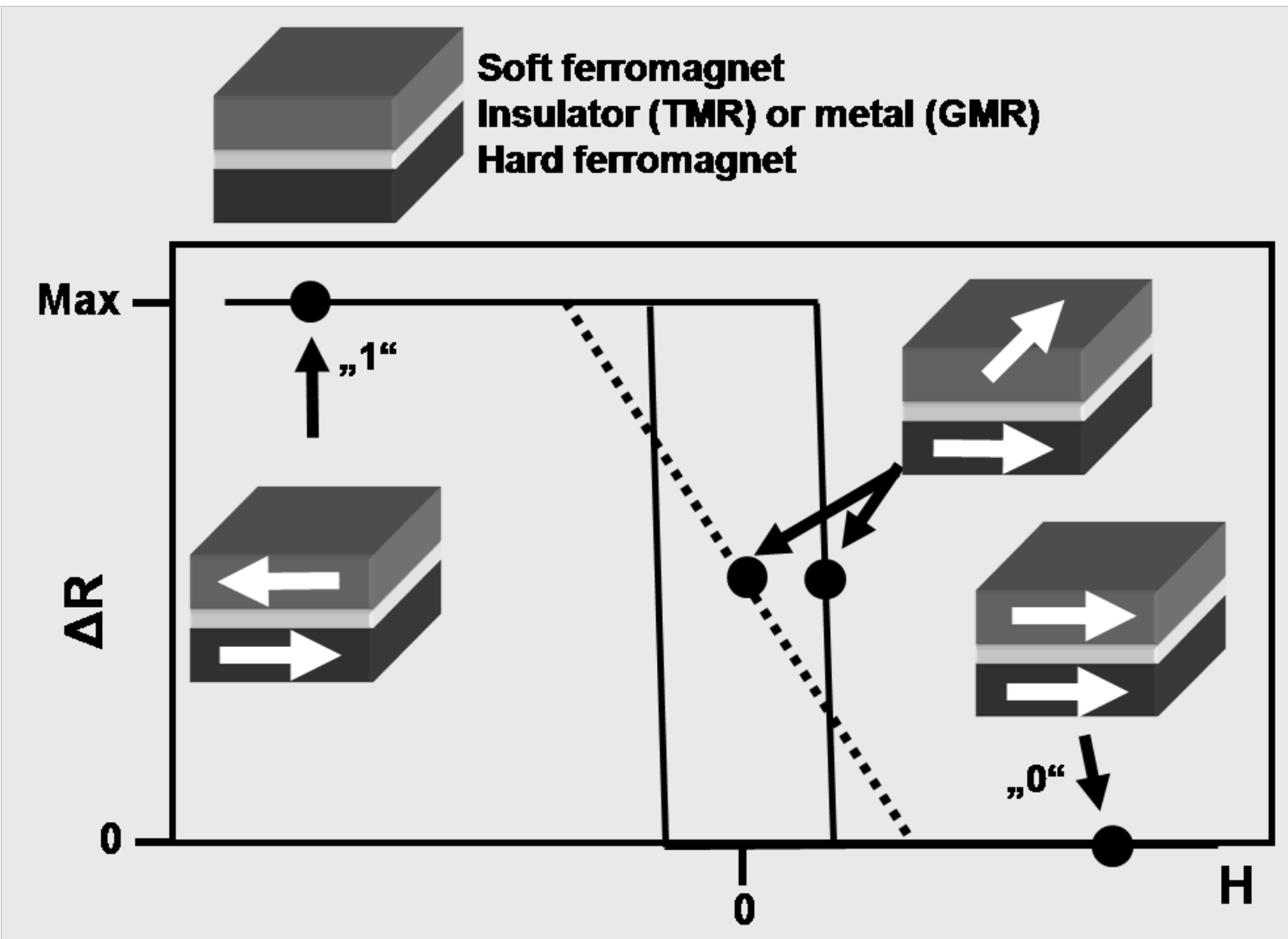

Figure 2: Typical responses of GMR- or TMR-devices to an external magnetic field $H$. The resistance increases when the alignement of the magnetizations changes from parallel $\left(, 0^{\star}\right)$ to antiparallel $\left(, 1^{\star}\right)$. The hysteresis (black line) mirrors that of the magnetization of the soft magnetic layer. Using modified film stacks, hysteresis free responses (dotted line) are also possible.

The physical reason for the resistance changes between the parallel and antiparallel magnetization states is the exchange splitting of the electronic density of states between electrons with majority and minority spin, respectively.

By tailoring the layer sequences of the sensors and their shape, different dependencies of the resistance on the external field can be realized. Whereas the linear dependence is appropriate for quantitative measurements of the external field, the hysteretic one can serve either for counting or for data storage. For the latter purpose, the high resistive state is usually identified with logic "1" and the lower with logic "O".

One major precondition for the applicability of GMR or TMR devices is their performance as compared to the major competing technologies, i.e. AMR- or Hall-devices. Typical resistance changes $\left(\Delta R_{\operatorname{Max}}\right)$ of, e.g., the established Anisotropic Magnetoresistance (AMR) are in the range of a few percent within a field range of some 10's of Oe with a very good signal to noise ratio. 
The first GMR-film stacks realized in the late 1980's already showed values of $\Delta R_{\text {Max }}$ of up to $60 \%$, but this effect amplitude was obtained only at high fields of some 1000's of Oe. The sensitivity defined as \% resistance change per applied Oe was, therefore, smaller than that of the established devices. By tailoring the materials of GMR and TMR-devices, however, the effect amplitude increased meanwhile up to some hundred $\%$ and the sensitivity up to some $10 \%$ resistance change per Oe, i.e. far beyond the limits of traditional devices.

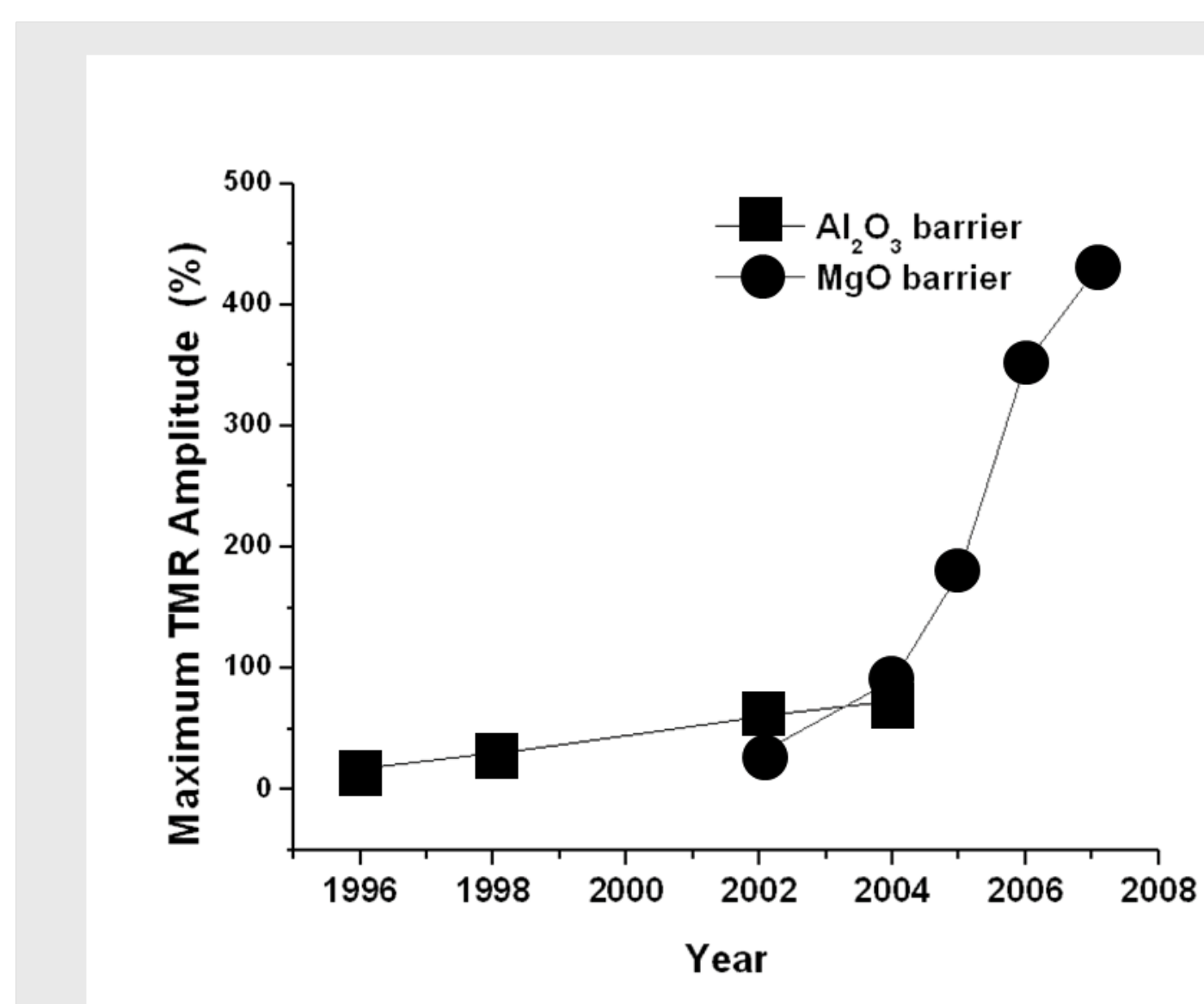

Figure 3: The maximum TMR amplitude reported from 1996 to 2008 for amorphous $\mathrm{Al}_{2} \mathrm{O}_{3}$ and crystalline MgO barriers. Major improvements have been achieved for a reliable device manufacturing process.

As an example, Figure 3 shows the development of the TMR amplitude at room temperature for the last decade. The new material MgO for the tunnel barrier gave a major breakthrough of the amplitude up to around $450 \%$ nowadays. This is based on the superior properties of these crystalline barriers in comparison with the amorphous $\mathrm{Al}_{2} \mathrm{O}_{3}$ material, giving rise to a "coherent tunneling" of the electrons [7].

\section{Device production}

The film stacks of magnetoelectronic devices are different if the Giant Magneto Resistance or the Tunneling Magneto Resistance are used. While the thicknesses of the indivídual layers range from about $0.8 \mathrm{~nm}$ to around $10 \mathrm{~nm}$ in each case, the layer stack for GMR is completely metallic and the sensing current is usually flowing in the film plane. For TMR, one insulating (usually oxidic) barrier layer must be inserted and the flow of the electric current is perpendicular to the film stack.

Usually, the film production is done by magnetron sputtering from several targets. Depending on the film stack for specific application, up to seven different materials are necessary. 


\section{Applications}

One major advantage of magnetoelectronics is the flexibility of the devices. Besides the possibility to tailor their response to a magnetic field (figure 2), also their resistance can be varied over more than 7 orders of magnitude. The shape of the response curve, i.e. the dependence of the resistance or the sensing current on the external magnetic field, can be tailored additionally as shown in figure 4 :

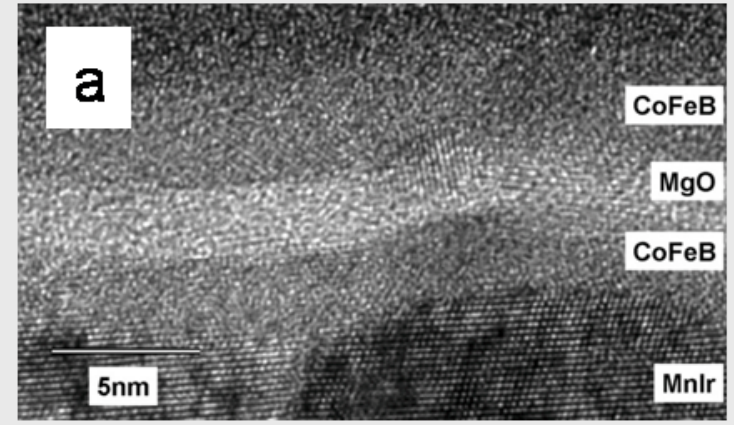

Microstructured TMR - sensors:

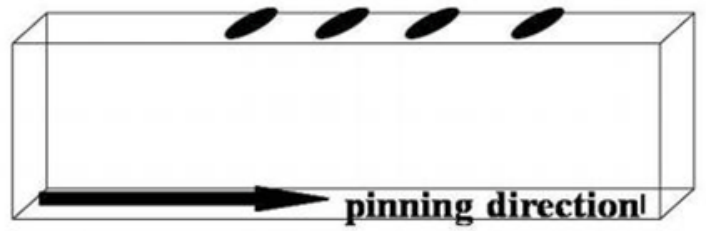

C Magnetic field
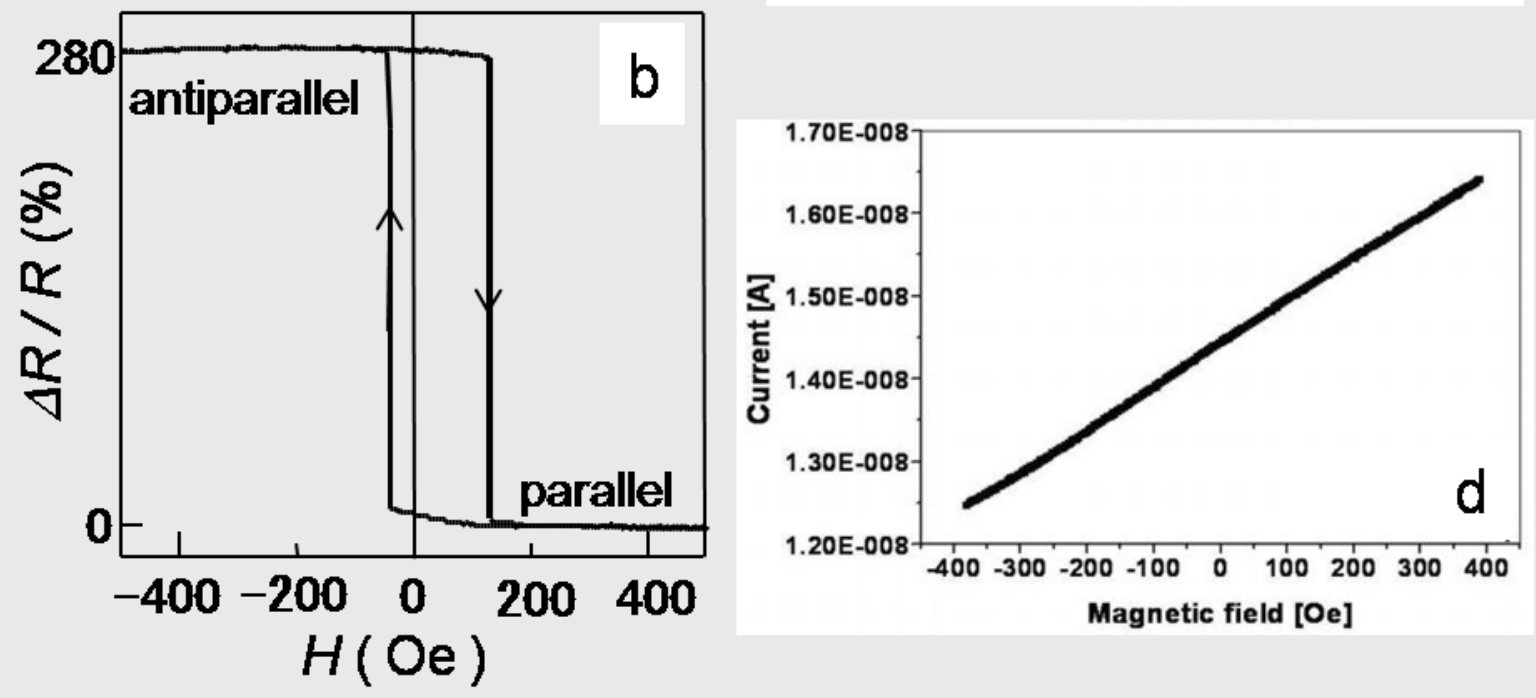

\section{Figure 4: A typical TEM cross section of a magnetic TMR sensor (a) and the resistance change as a function of a magnetic field (b). Part (c) shows the device layout for a linearization of the resistance vs field curve; in (d), the linearized sensing current for a CoFeB I Mgo / CoFeB sensor is displayed as a function of the external field.}

Here, a TEM crossection of a TMR sensor (fig. 4a) based on CoFeB / MgO / CoFeB, which was annealed at $375^{\circ} \mathrm{C}$ prior to the imaging, shows both the typical almost atomic precision of the device structure as well as the stability against high temperatures: Most of the TMR sensor types can withstand temperatures up to $400{ }^{\circ} \mathrm{C}$. In fig. $4 \mathrm{~b}$, a typical "minor loop" of the resistance vs. field curve mirrors the hysteresis of the upper CoFeB ferromagnetic electrode layer. In these stacks, the lower electrode is magnetically fixed by direct contact to a natural antiferromagnet (IrMn in this example). Such hysteretic response curves can be used for data storage, data processing and counters (for, e.g., pole wheels).

Many applications, however, require not only counting field changes, but also measuring quantitatively the strength of an external magnetic field. For this, one can use the shape anisotropy in order to reduce the hysteresis of the response and to linearize the curve. In order to obtain a linear dependence of the sensing current on the magnetic field, as shown in fig. 4c, the lower ferromagnetic electrode is fixed by the pinning to the antiferromagnet. Then, the upper electrode is patterned to an ellipsoidal shape with the long axis perpendicular to the direction of the pinning, i.e. to the magnetization of the lower electrode. 
Thus with zero external field, the magnetizations of lower and upper electrode are perpendicular. An external field will now turn the magnetization of the upper electrode from the long axis direction of the ellipsoid, which is a magnetic easy axis, towards the short (hard) axis. This results in a vanishing hysteresis of the magnetization process and thus the anticipated linear dependence of the sensing current on the external field can be obtained (fig. 4d).

Due to their large signal and temperature stability, GMR- or TMR sensors do have the potential to replace the commonly used AMR- or Hall sensors in typical industrial applications such as position measurements or angle detection.

There are, however, some new fields evolving for these sensors:

- Medical diagnostics: For measuring, e.g., the heart or brain activity with some spatial resolution, SQUID sensors are used to detect the magnetic field fluxes generated by the electric currents related with the respective activities. The sensitivity needed for this measurement is, however, much larger than for traditional applications, because it is necessary to detect magnetic flux density strengths of pico- to femtoTesla. Therefore, the noise level of the sensors becomes a major issue. Here, SQUIDs reach a level of around $3 \mathrm{fT} / \mathrm{sqrt}(\mathrm{Hz})$ at liquid Helium temperature $(4.2 \mathrm{~K})$. GMR sensors turned out to be the most promising candidates for competing with SQUIDs, because they can reach low noise levels $(10 \mathrm{fT} / \mathrm{sqrt}(\mathrm{Hz}))$ at the temperature of liquid Nitrogen $(77 \mathrm{~K})$ [8], Thus first prototypes of sensor arrays for imaging the heart and brain activities are currently being tested.

- Biotechnological applications: In biotechnology, molecules like DNA or proteins are frequently marked by magnetic spheres called 'beads'. This opens the possibility to measure the presence or absence of these biomolecules by detecting the magnetic beads with a GMR or TMR sensor. This technique was first developed at the Naval Research Labs using Giant Magnetoresistance sensors [9]. Simultaneously, the development of homogeneous magnetic nanoparticles with sizes between some nm and $100 \mathrm{~nm}$ gave the possibilty, to adjust the sizes of the magnetic markers to those of the biomolecules to be detected [10]. Thus the task of the sensors is to reliably measuring a small number of magnetic particles deposited on top of the sensor surface.
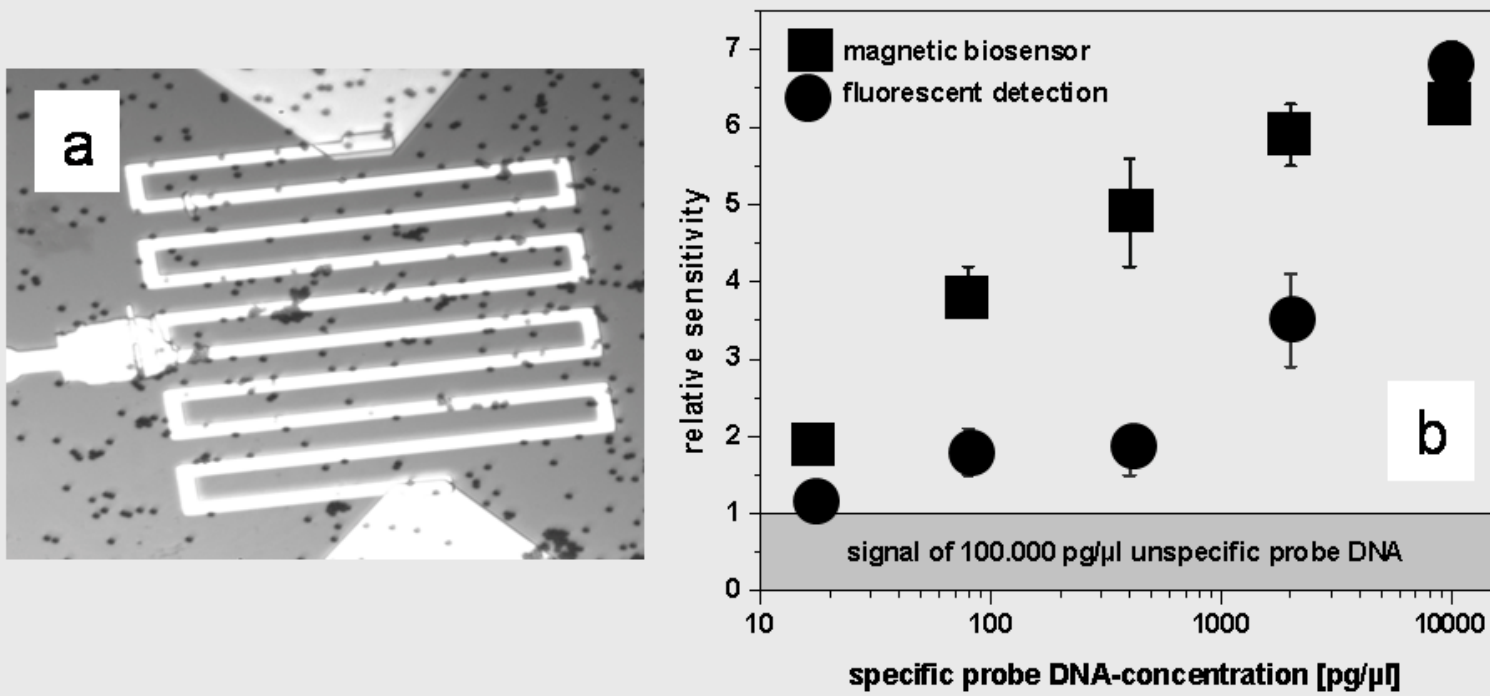

Figure 5: A typical GMR meander shaped sensor for the detection of small magnetic particles deposited on top of the sensor surface (a) and the resulting signal (relative sensitivity) as compared to standard fiuorescent detection of the DNA molecules.

Figure 5 shows in (a) the layout of a GMR type sensor for these measurements, which is already covered by magnetic particles with a density of 0.04 particles $/ \mu \mathrm{m}^{2}$. In (b), the GMR sensor signal is compared with the signal of a standard fluorescent detection technique as a function of DNA concentration [11]. 
As can be seen in Figure 5, reasonable signals as in dependence of the concenbtration of DNA (and in turn of magnetic particles) are obtained. The direct comparisons with established optical methods of marking DNA with fluorescent molecules showed, that the magnetic biosensor can be more sensitive at low concentrations of the analyte molecule, which is the most interesting area for their application.

\section{Summary}

Within the new field of Spin- or Magnetoelectronics basic discoveries emerged rapidly to a new technology for both niche as well as mass markets. While sensors are alreadyapplied as read heads in hard disk drives solid state memories such as a Magnetic Random Access Memory (MRAM) or logic gates are still under development.

For sensor applications, however, these new devices offer both larger signal amplitude as well as a good temperature stability. Moreover, the output signal of the sensors can be tailored by changing the materials and the sensor layout. Both hysteretic as well as linearized signals can be obtained and used for, e.g., counting or quantitative measurements of the strength of a magnetic field. At the same time, new field are emerging in medical and biotechnological applications: Imaging of heart of brain activities becomes possible by optimizing the noise level in all metallic GMR devices and the detection of biomolecules marked by magnetic particles was demonstrated.

The further development now concentrates on integrated systems, i.e. manufacturing sensors as well as small capacity memories on one chip and one technology platform. Moreover, arrays of these sensors could provide new opportunities in, e.g., imaging complex structures containing magnetic quantities or tracking the movement of magnetic particles.

\section{References}

[1] P. Grünberg, R. Schreiber, Y. Pang, M.B. Brodsky, and H. Sowers, Phys. Rev. Lett. 57 (1986)

[2] M.N. Baibich, J.M. Broto, A. Fert, F. Nguyen van Dau, and F. Petroff, Phys. Rev. Lett. 61 (1988)

[3] J.S. Moodera, L.R. Kinder, T.M. Wong, and R. Meservey, Phys. Rev. Lett. 74 (1995)

[4] T. Miyazaki and N.J. Tezuka, J. Magn. Magn. Mater. 139 (1995)

[5] G.A. Prinz, Science 282 (1998)

[6] Press release of the swedish royal academy of science, http://nobelprize.org/nobel_prizes/physics/laureates/2007/index.html

[7] G. Reiss, J. Schmalhorst, A. Thomas, A. Hütten, S. Yuasa, Magnetic Tunnel Junctions, Springer Verlag, Eds. Hartmut Zabel and Samuel Bader, Springer Tracts in Modern Physics 227 (2008) 291-333

[8] M. Pannetier-Lecoeur, C. Fermon, A. de Vismes, E Kerr, L. Vieux-Rochaz, J. Magnet. Magnet. Mater. 316 (2007) E246

[9] D.R. Baselt, G.U. Lee, M. Natesan, S.W. Metzger, P.E. Sheehan, and J.R. Colton, Biosens.

Bioelectron. 13 (1998)

[10] A. Hütten, D. Sudfeld, I. Ennen, G. Reiss, K. Wojczykowski, P. Jutzi, J. Magnet. Magnet. Mater. 293 (2005) 93

[11] J. Schotter, M. Panhorst, M. Brzeska, P.B. Kamp, A. Becker, A, Pühler, G. Reiss, H. Brückl, Molecular Detection with Magnetic Labels and Magnetoresistive Sensors, In Nanoscale Devices Fundamentals and Applications, Springer 2006 (R. Gross et. al. edt.) pp 35 - 46 